\title{
STABILITY OF LIQUID FILMS DOWN AN INCLINED PLANE
}

\author{
Phan Thi Thu Phuong and Tran Van Tran \\ Hanoi University of Natural Sciences \\ Vietnam National University, Hanoi
}

\begin{abstract}
In this paper, the problem of linear stability of viscous liquid films down an inclined plane is solved by finite difference method. It is applicable for moderate values of Reynolds and wave numbers. The obtained results by this method is compared with ones of some papers and with experimental data.
\end{abstract}

\section{INTRODUCTION}

The flow of liquid films down an inclined wall has been often met in Nature, in chemical and food processing industries. This kind of flows may be also observed when very hot solid surfaces need to be cooled safely and effectively. The problem of linear stability of liquid films on inclined plane has been studied early from the work of Kapitza [2]. In later works of many authors, the problem was solved for long wave disturbances at small Reynolds numbers by seeking the solution in some kinds of series. For vertical films as shown by experiments and by some theoretical works, instability may occur at very low Reynolds numbers.

In last decades of the previous century, nonlinear stability of liquid films down an inclined plane as well as linear stability of heated inclined liquid films had been investigated intensively.

The purpose of this paper is to take attempt to solve the stability problem for flows with free surface by numerical method. It is expected that this method will be useful in solving more complicated problem related to flows of the above mentioned kind.

\section{PROBLEM DESCRIPTION}

A viscous liquid layer of thickness $\mathrm{H}$ on a solid surface with an inclination angle $\theta$, flows down under the gravity action. We call this undisturbed laminar uniform two-dimensional flow as a basic, whose stability is considered here. We also suppose that the liquid film is bounded by a gas on the free surface so that the action of surface tension is available there. According to [1], we can get the problem in the eigenvalue form:

$$
\begin{gathered}
\left(\varphi^{(4)}-2 \alpha^{2} \varphi^{\prime \prime}+\alpha^{4} \varphi\right)-i \alpha \operatorname{Re}\left[(U-c)\left(\varphi^{\prime \prime}-\alpha^{2} \varphi\right)-U^{\prime \prime} \varphi\right]=0 \\
\varphi(0)=\varphi^{\prime}(0)=0 \\
\varphi(1)=\varsigma(c-1) \\
\alpha^{2} \varphi(1)+\varphi^{\prime \prime}(1)-2 \zeta=0
\end{gathered}
$$




$$
\varphi^{\prime \prime \prime}(1)-\left[3 \alpha^{2}+i \alpha \operatorname{Re}(U-c)\right] \varphi^{\prime}(1)-i \alpha \operatorname{Re} \zeta S=0
$$

where $\alpha$ is wave number, $R e$ is Reynolds number, $\zeta$ is a constant, and $\varphi$ is stream function.

while

$$
S \equiv \frac{\cos \theta}{F r}+G \alpha^{2}
$$

$$
G=\frac{\sigma}{\rho}(g \sin \theta)^{-1 / 3}\left(\frac{\mu}{\rho}\right)^{-4 / 3}
$$

with Fr is Froude number, $\rho$ is liquid density, $\mu$ is dynamic viscosity of the liquid, $g$ is the gravity acceleration and $\sigma$ is perturbation of pressure.

$c=c_{r}+i c_{i}$ is, in general, complex, the sign of $c_{i}$ defines stability of the basic flow, namely if $c_{i}>0$, then the flow is linearly unstable. In the opposite case, the flow is regarded linearly stable. Value $c_{r}$ expresses non-dimensional propagation velocity of waves, while value $\alpha c_{i}$ is called time increment.

Equation (2.1) is well known as the Orr-Sommerfeld equation for linear stability of two-dimensional parallet flows. Solution of the above problem from the linear stability point of view is to determine eigenvalue $c$ at any given pair of values Re and $\alpha$. The curve on the plane $(\alpha, R e)$ along which one has $c_{i} \equiv 0$ is called as the neutral curve that is of particular interest.

\section{METHODS OF SOLUTION}

The problem $(2.1) \div(2.5)$ has been theoretically and experimentally studied by many authors. For theoretical works one can mention papers of $[1,2,3]$. Experimental data are given in works of $[2,3]$. It should be noted that, in some listed above works, the vertical axis was chosen on opposite direction to that given here, and also the origin of coordinate system was located at the free surface of the liquid. Of course, these differences in notation do not influence the stability nature of the problem.

In previous works, the authors often represented the solution in power series of $y$ coordinate and restricted by several first numbers. As expected, this approach has been valid for small values of Re and $\alpha$. In this paper we use finite difference method (FDM) to solve the stability problem without any restriction on wave numbers and moderate Reynolds numbers. It seems to us that there may be two reasons why FDM so far has not been used for this problem. First, as observed in experiments vertical liquid films are unstable at low Reynolds number, and often long waves have been observed on the free surface. Second, the existence of the third derivative in (2.5) causes some difficulties in implementing FDM for this problem. However, as we will see later, not vertically inclined films lose their stability at moderate Reynolds number, so solution of the stability problem at those Reynolds numbers is still desirable.

In order to apply finite difference method we use (2.3) to rewrite (2.4) and (2.5) respectively in the form:

$$
\begin{gathered}
\varphi^{\prime \prime}(1)+\left(\alpha^{2}-\frac{2}{c-1}\right) \varphi(1)=0 \\
\varphi^{\prime \prime \prime}(1)-\left[3 \alpha^{2}+i \alpha \operatorname{Re}(U-c)\right] \cdot \varphi^{\prime}(1)-i \alpha \operatorname{Re} S \frac{\varphi(1)}{c-1}=0,
\end{gathered}
$$


Thus, our eigenvalue consists from (2.1), (2.2), (3.1) and (3.2). Follow Thomas (1953)[4], here we will use modified central five-point schemes of higher accuracy for $\varphi^{\prime}, \varphi^{\prime \prime}$ and $\varphi^{(4)}$ by introducing a complementary function $g^{\prime}(y)$ by the formula:

$$
\varphi\left(y_{i}\right)=\left(1+\frac{\delta^{2}}{6}+\frac{\delta^{4}}{360}\right) g\left(y_{i}\right)
$$

Then one can have:

$$
\begin{gathered}
\varphi^{\prime}\left(y_{i}\right)=\frac{1}{h} \chi g\left(y_{i}\right)+O\left(h^{4}\right) \\
\varphi^{\prime \prime}\left(y_{i}\right)=\frac{1}{h^{2}}\left(\delta^{2}+\frac{\delta^{4}}{12}\right) g\left(y_{i}\right)+O\left(h^{6}\right) \\
\varphi^{(4)}\left(y_{i}\right)=\frac{1}{h^{4}} \delta^{4} g\left(y_{i}\right)+O\left(h^{4}\right)
\end{gathered}
$$

where $h=\frac{1}{N}$ is the step of a uniform net of integration from $N+1$ net points including two boundaries $\mathrm{y}=0$ and $\mathrm{y}=1$. Formulae $(3.3) \div(3.6)$ are written at net point $y_{i}=(i-1) h$ with the following notations:

$$
\begin{gathered}
\delta^{2} g\left(y_{i}\right)=g\left(y_{i}-h\right)-2 g\left(y_{i}\right)+g\left(y_{i}+h\right) \\
\delta^{4}=\delta^{2}\left(\delta^{2}\right) \\
\chi \chi g\left(y_{i}\right)=\frac{1}{2}\left[g\left(y_{i}+h\right)-g\left(y_{i}-h\right)\right]
\end{gathered}
$$

Unfortunately, for $\varphi^{\prime \prime \prime \prime}$ one cannot increase the accuracy by (3.3), so we have to use scheme

$$
\varphi^{\prime \prime \prime}\left(y_{i}\right)=\frac{1}{h^{3}} \delta^{2}\left(\chi g\left(y_{i}\right)\right)+O\left(h^{2}\right)
$$

However, the imperfection of (3.7) is not serious in this case because the problem has been solved at moderate Reynolds numbers only, and it may be corrected by taking suitable step of integration $h$.

Now applying (3.3), (3.7) to the equation (2.1), and to condition (2.2), (3.1), and (3.2), one obtains a system of linear equations of the form

$$
A_{k} g_{k-2}+B_{k} g_{k-1}+C_{k} g_{k}+D_{k} g_{k+1}+E_{k} g_{k+2}=0(k=1 \div N+1)
$$

Note that, for approximating equation (2.1) and boundary condition at $y_{1}=0$ and $y_{N+1}=1$ one has to introduce two fictitious points on the left of point $y_{1}$, and two ones on the right of point $y_{N+1}$ with the same step $h$. Next, using finite difference equations for (2.2) we manipulate equation (3.8) at points $y_{1}$, and $y_{2}$ to the following forms respectively:

$$
\begin{gathered}
\bar{C}_{1} g_{1}+\bar{D}_{1} g_{2}+\bar{E}_{1} g_{3}=0 \\
\bar{B}_{2} g_{1}+\bar{C}_{2} g_{2}+\bar{D}_{2} g_{3}+\bar{E}_{2} g_{4}=0
\end{gathered}
$$

Analogously, one can reduce equation (2.1) at points $y_{N}$ and $y_{N+1}$ respectively to the forms:

$$
\begin{aligned}
& \bar{A}_{N} g_{N-2}+\bar{B}_{N} g_{N-1}+\bar{C}_{N} g_{N}+\overline{\bar{D}}_{N g_{N+1}}=0 \\
& \bar{A}_{N+1} g_{N-1}+\bar{B}_{N+1} g_{N}+\bar{C}_{N+1} g_{N+1}=0
\end{aligned}
$$


where the over bar in $(3.9) \div(3.12)$ shows that those values are results of some algebraic manipulations. The system of equations consisting of $(3.9) \div(3.12)$ and (3.8) with $k=3 \div N-1$ is a standard type of a modified Thomas system. As a rule, the characteristic equation for determining eigenvalue $c$ has been obtained from the requirement that this system has a nontrivial solution that in turn reduces to condition that its determinant should be equal to zero.

Consider a procedure of getting the characteristic equation relying on the modified Thomas algorithm. Equations of the above Thomas system are easy to reduce each to the form

$$
g_{k}+G_{k} g_{k+1}+H_{k} g_{k+2}=0 \quad k=1 \div N
$$

where coefficients $G_{k}$ and $H_{k}$ are calculated by recurrent formulae from $k=1$ to $k=N$. form

Using (3.13) with $k=N-1$ and $k=N\left(\mathrm{H}_{N}=0\right.$ for $\left.k=N\right)$ to transform (3.12) to the

$$
F g_{N+1}=0
$$

where

$$
F(\alpha, R e, c)=\bar{A}_{N+1}\left(G_{N-1} G_{N}-H_{N-1}\right)-\bar{B}_{N+1} G_{N}+\bar{C}_{N+1}
$$

Then the characteristic equation simply is

$$
F=0
$$

Here root of this equation is calculated by Newton method for every given pair of values $\operatorname{Re}$ and $\alpha$.

\section{RESULTS AND DISCUSSION}

On the table below, the neutral stability was calculated by two methods for vertical water films at $19^{\circ} \mathrm{C}$ that makes $S=3300$. The results obtained by FDM and by formula (4.13) in [1] are presented in column 2 and column 3 of the table respectively.

As shown on the table, for very long waves (very small $\alpha$ ), the results obtained by FDM are less sensitive in comparison with ones of the Benjamin's method. It may relate to the fact, that the integration step $h$ is not small enough.

Except these very long disturbances, we have a good agreement between parameters of the neutral curve calculated by finite difference method and those given by (4.13) of Benjamin paper [1]. The agreement is satisfactory even up to a limit Reynolds number of Benjamin's method that is around 41.5 for vertical water at film $19^{\circ} \mathrm{C}$ (see the second and the third columns of the table). The solution of linear stability problem by finite difference method also completely supports the Benjamin's disclosure that there is no critical Reynolds number for vertically falling liquid films.

As a rule, finite difference method described here has no restriction on both Reynolds and wave number for problem $(2.1) \div(2.5)$. So for the aim of reference neutral stability for vertical films was calculated beyond the Benjamin's critical Reynolds number up to 100. These results are given in the second column of the table.

The results for some vertical films obtained here by finite difference method have been compared also with experimental data given in [3] on Fig. 1. The agreement is good enough both qualitatively and quantitatively. 
Table 1. Parameters of the neutral curve for vertical water film

\begin{tabular}{|c|c|c|}
\hline \multirow{2}{*}{$R e$} & \multicolumn{2}{|c|}{ Wave number $(\alpha)$} \\
\cline { 2 - 3 } & Finite Difference Method & Benjamin's formula \\
\hline 0.001 & 0.0005 & 0.0001 \\
\hline 0.005 & 0.0005 & 0.0004 \\
\hline 0.010 & 0.0005 & 0.0006 \\
\hline 0.021 & 0.0005 & 0.0011 \\
\hline 0.026 & 0.0005 & 0.0013 \\
\hline 0.030 & 0.0005 & 0.0015 \\
\hline 0.038 & 0.0005 & 0.0018 \\
\hline 0.041 & 0.0018 & 0.0019 \\
\hline 0.045 & 0.0019 & 0.0021 \\
\hline 0.050 & 0.0022 & 0.0023 \\
\hline 0.100 & 0.0041 & 0.0040 \\
\hline 0.500 & 0.0156 & 0.0154 \\
\hline 1.000 & 0.0277 & 0.0275 \\
\hline 2.000 & 0.0492 & 0.0488 \\
\hline 3.000 & 0.0697 & 0.0682 \\
\hline 4.000 & 0.0869 & 0.0862 \\
\hline 5.000 & 0.1041 & 0.1033 \\
\hline 10.000 & 0.1792 & 0.1778 \\
\hline 20.500 & 0.3010 & 0.2983 \\
\hline 30.500 & 0.3943 & 0.3941 \\
\hline 40.500 & 0.4742 & 0.5230 \\
\hline
\end{tabular}

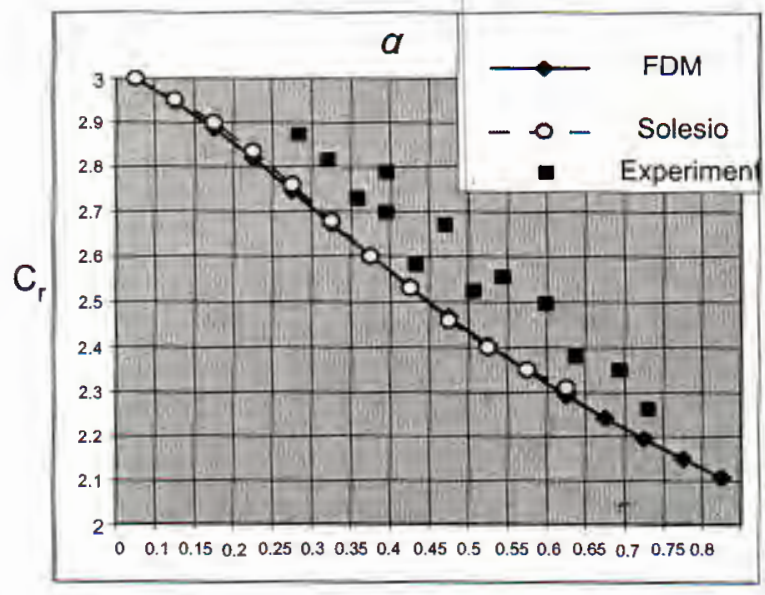

a)

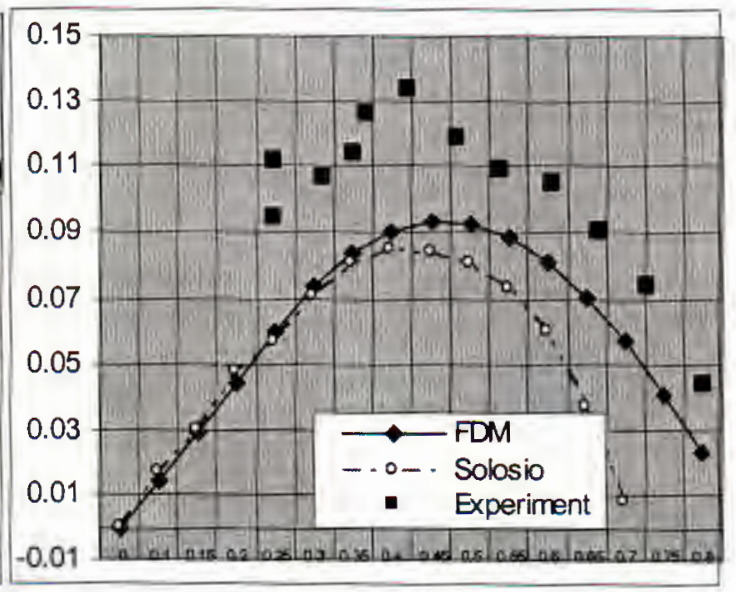

b)

Fig. 1. a) Wave velocity comparision. b) Time increment comparision 


\section{REFERENCES}

1. T. B. Benjamin, Wave formation in laminar flow down an inclined plane, J. Fluid Mechanics 2 (1957) 554-574.

2. P. L. Kapitza, Wave motion in thin layers of viscous fluid, J. Exp. Theor. Physics, USSR, 18 (3) (1949) 105.

3. J. N. Solesio, Critères de stabilité d'un film liquide s'écoulant sur plan incliné, C.R. Acad. Sc. Paris, 282 (10) (1976) 539-542.

4. L. H. Thomas, The stability of plane poiseuill flow, Phys. Rev. 91, 780.

Received October 07, 2005

Received May 05, 2006

\section{ỔN DỊNH CỦA DÒNG CHẢY CHẤT LÒNG TRÊN MẶT PHĂNG NGHIÊNG}

Trong bài báo này, vấn đề ổn định tuyến tính của dòng cháy hai chiều trên mặt phẳng nghiêng được nghiên cứu bằng phương pháp sai phân hữu hạn với độ chính xác nâng cao. Phương pháp này có thể dùng được cho mọi giá trị trung bình của số Reynolds và số sóng. Kết quả đạt được bằng phương pháp này được so sánh với kết quà của một số bài báo khác cũng như kết quá thực nghiệm. 